\title{
Case report on Textiloma of Uterus
}

\author{
RAHIMA BEGUM ${ }^{1}$, SHAKILA KHANUM ${ }^{2}$
}

\begin{abstract}
:
A 30years of old hypothyroid lady, presented with foul smelling excessive whitish per vaginal discharge, associated with itching in vulva \& pain in the lower abdomen following caesarean section 3 months back. Her uterus was palpably enlarged and firm. USG revealed bulky uterus with wall echo shadow sign. A CT scan of whole abdomen was done that revealed intrauterine textiloma. During laparotomy huge amount of pus came out through wound and a mob was found within uterine cavity. Textiloma is a rare problem of surgery arising from retained gauze during operation. Careful counting of surgical gauze before closure and identification by newer techniques is required to prevent this iatrogenic complication.
\end{abstract}

\section{Introduction:}

Textiloma is the technical term, derived from latin" textile", a woven febric and the suffix "-oma", meaning a tumor or swelling. It is a historical term reffered to pseudo tumor formation and inflammatory reaction caused by a foreign body or retained non absorbable cotton matrix left behind mistakenly in patient body. They usually caused by abdominal or gynaecological surgery.

The incidence of Textiloma is difficult to calculate as some patients remain asymptomatic and are never discovered. This condition is often underestimated because case numbers are calculated only on the basis of malpractice claims. Another reason of underreporting of occurrences is due to the fear of medicolegal repercussions.

\section{Case report:}

Mrs. X, 30years of age, known hypothyroid, presented with foul smelling excessive whitish per vaginal discharge, associated with itching in vulva \& pain in the lower abdomen following caesarean section 3 months back.

On examination, the patient is mildly anemic, obese with BMI - 30 and normal vital parameters. Genital examinations revealed cervix is congested; uterus is 18 weeks size \& firm in consistency. Other local and systemic examinations were normal.

Investigations revealed, $\mathrm{Hb}-10.50 \mathrm{gm} / \mathrm{dl}$, TC of WBC $-12,40 / \mathrm{cmm}$, DC of WBC - 67\%-25\%-5\%-3\%, ESR$90 \mathrm{~mm}$ in Ist hour. Thyroid function was normal. Urine R/E \& C/S, Blodd C/S and High vaginal swab showed no growth. USG of WIA showed - Uterus was bulky, Endometrial thickness is $1.52 \mathrm{~cm}$; wall echo shadow sign is noted in uterine endometrium, Echotexure of uterine myometrium is normal. Cervical cytology was negative for malignancy, but there was severe inflammation. Colposcopic biopsy was taken which showed acute \& chronic cervicitis with squamous metaplasia and mild dysplasia(CIN-I). A CT scan of whole abdomen was done that revealed intra-uterine textiloma and -chronic cystitis.

So decision was taken to do laparotomy. Abdomen was opened by pfannenstiel incision. Omentum was found densely adherent with parietal peritoneum. The

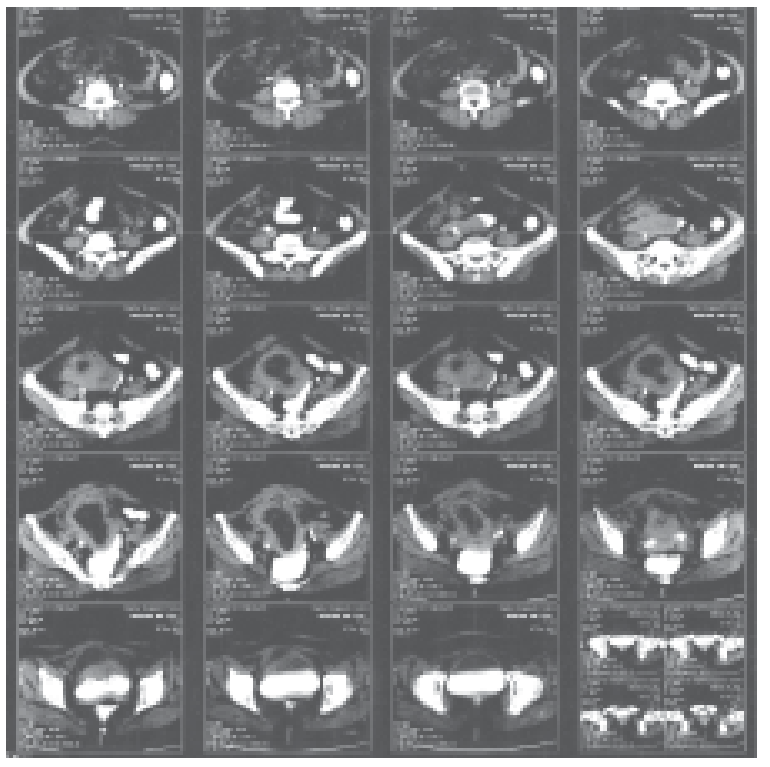

1. Head of Department of Obstetrics \& Gynecology, BIRDEM

2. Medical Officer, Department of Obstetrics \& Gynecology, BIRDEM 


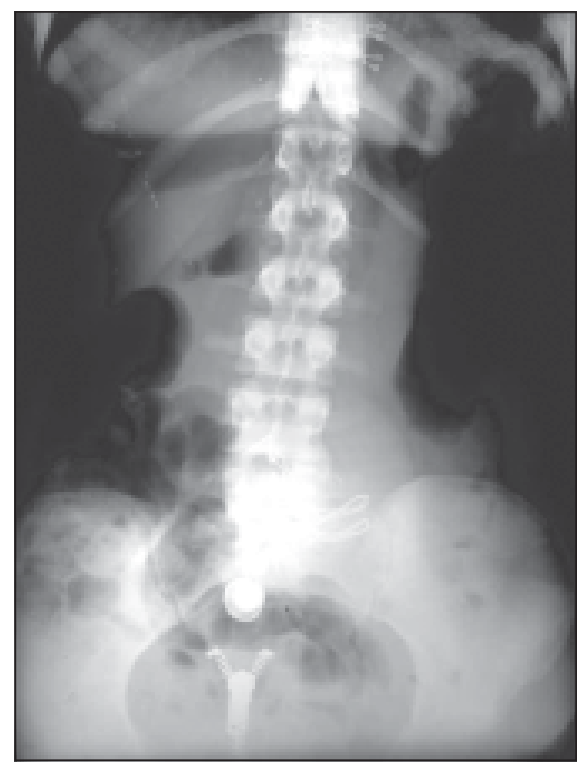

Plain X-ray of the abdomen showing the opaque marker of the retained gauze in the center of abdomen

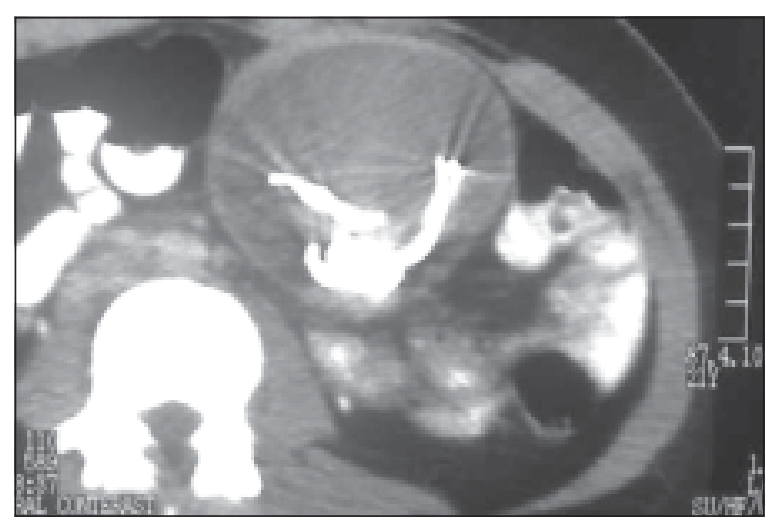

Abdominal CT scan showing a round well-defined softtissue mass containing an internal high-density area in the mid-abdomen

adhesions were separated. Anatomy of pelvis and lower abdomen was distorted. Uterus was burried in adhesion. Previous scar of C/S was opened during mobilization and huge amount of pus came out through wound and a mob was found within uterine cavity. Mob removed and TAH and BSO were done. Post operative period was uneventful and patient was discharged after recovery.

\section{Discussion:}

Despite all considerations during operation, retained gauzes are still one of the major problems. Textiloma or gossypiboma usually occurs in one out of 100 to 3000 of all surgical investigations and one out of 100015000 intra abdominal operations. The incidence of retained foreign bodies following surgery has a reported

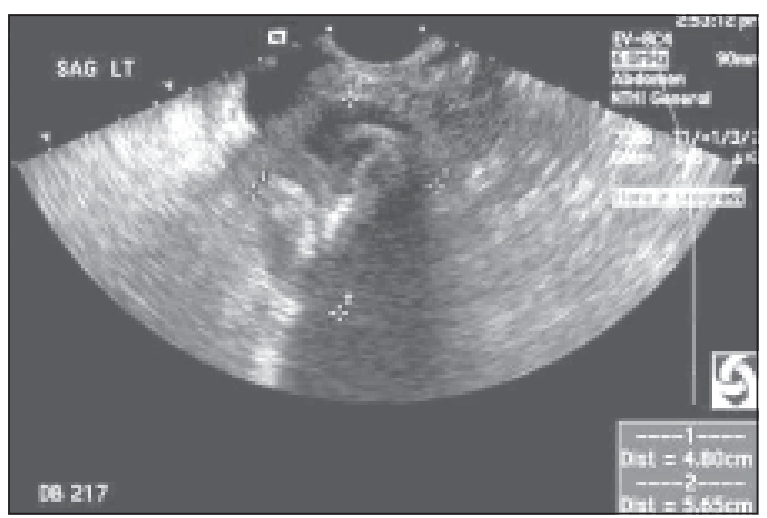

Ultrasound images in the area of the radio-adnexa show curvilinear areas of high echogenicity within a mass

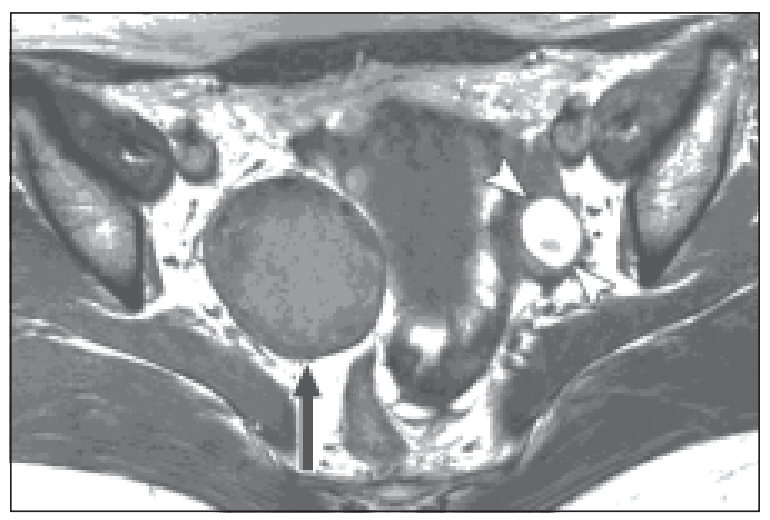

Axial T1-weighted image shows well-defined mass with heterogeneously high signal in central cavity and low signal in peripheral wall.

rate of $0.01 \%$ to $0.001 \%$, of which Textilomas make up $80 \%$ of cases. ${ }^{1}$

Because the symptoms of Textiloma are usually nonspecific, depends on the location of the sponge and the type of reaction and may appear years after surgery, The diagnosis of Textiloma usually comes from imaging studies and a high index of suspicion. Common symptoms and signs of Textiloma are abdominal distention, tenesmus, pain, palpable mass, vomiting, weight loss, diarrhea, abscess, and fistula formation.

Surgical sponges are made of cotton that does not stimulate any specific biochemical reaction except adhesion and granuloma formation. They may be a cause of an asymptomatic condition for a long time. 
Textiloma can have two different types of body responses: exudative and aseptic fibrous. The latter can have adhesions, encapsulation, and, eventually, granuloma formation. However, the former usually occurs early in the postoperative period and may involve secondary bacterial contamination, which results in various fistulas. ${ }^{2}$

Possible causatives of sponge retention are emergency surgery, unexpected change in the surgical procedure, disorganization, hurried sponge counts, long operations, unstable patient condition, inexperienced staff, inadequate staff numbers and patient with high body mass index (BMI). Of these risk factors, the most significant risk factors are emergency surgery, unplanned change in the operation and BMI. There is 9-fold increase in risk associated with emergency surgery.

Textilomas most commonly occur in the abdominal or pelvic cavity, as after gynecologic and upper abdominal surgical procedures. Much of the Textilomas (75\%) are identified only after abdominal or pelvic surgery. Textiloma has also been reported after - neurosurgery (Intracranial, spinal), chest surgery, maxillofacial surgery.

Retained surgical sponges can cause serious consequences such as - bowel or visceral perforation, obstruction or fistula formation, sepsis or even death. Intra-abdominal Textilomas can migrate into the ileum, stomach, colon or bladder without any apparent opening in the wall of these luminal organs. It is difficult to recognize a Textiloma by using radiological screening if the sponge does not have any radiological marker on itself, because the cotton can simulate hematoma, granulomatous process, abscess formation, cystic masses or neoplasm. Textiloma can have atypicall calcification and air bubbles as well. Many radiologic findings are characteristically used to diagnose Textiloma. ${ }^{3}$ If the sponge contains a radiopaque marker, the diagnosis can be made easily by conventional radiography. However, the retained sponge might not be identified at conventional radiography if a radiopaque sponge breaks into pieces or if the marker becomes bound or folded. The sonographic findings are a well-defined mass with a wavy hyperechoic area and dense acoustic shadowing. On CT scans, a retained sponge is typically seen as a well-defined soft-tissue mass and may show a whorled texture or a spongiform pattern with contained gas bubbles. Calcification of the wall of the mass may be observed on CT scans. ${ }^{4}$

To prevent Textiloma, sponges are counted by hand before and after surgeries. This method was codified into recommended guidelines in the 1970s by the Association of periOperative Registered Nurses (AORN). ${ }^{5}$
Four separate counts are recommended: the first when instruments and sponges are first unpackaged and set up, a second before the beginning of the surgical procedure, a third as closure begins, and a final count during final skin closure. In most countries, surgical sponges contain radiopaque material that can be readily identified in radiographic and CT images, facilitating detection. Some surgeons recommend routine postoperative X-ray films after surgery to reduce the likelihood of foreign body inclusion. Newer technologies are being developed that will hopefully decrease the incidence of retained foreign body, like radiofrequency identification (RFID). ${ }^{6}$ In this technology, commonly used surgical gauze sponges, which have been tagged with a radiofrequency identification (RFID) chip scanned with a barcode scanner.

\section{Conclusion:}

Textilomas are uncommon, mostly asymptomatic, and hard to diagnose. Particularly chronic cases do not show specific clinical and radiological signs for differential diagnosis. Textiloma should be included in the differential diagnosis of soft-tissue masses detected in patients with a history of a prior operation. Patient-clinician and clinician-radiologist interactions and compliance enhance the possibility of accurate diagnosis.

\section{References:}

1. Dane C, Yayla M, Dane B. A foreign body (gossypiboma) in pregnancy: first report of a case. Gynecol Surg. 2006;3(2):130-1.

2. Kaiser CW, Friedman S, Spurling KP, Slowick $\mathrm{T}$, Kaiser HA. The retained surgical sponge. Ann Surg 1996;224 : 79-84

3. Lu YY, Cheung YC, Ko SF, Ng SH. Calcified reticulate rind sign: a characteristic feature of gossypiboma on computed tomography. World J Gastroenterol 2005

4. Naik KS, Carrington BM, Yates W, Clarke NW. The post-cystectomy pseudotumour sign: MRI appearances of a modified chronic pelvic haematoma due to retained haemostatic gauze. Clin Radiol2000; 55:970 -974

5. Shyung LR, Chang WH, Lin SC, Shih SC, Kao CR, Chou SY (February 2005). "Report of gossypiboma from the standpoint in medicine and law". World J. Gastroenterol. 11 (8): 1248-9.

6. Gibbs VC, Coakley FD, Reines HD. Preventable errors in the operating room: retained foreign bodies after surgery. Curr Probl Surg. 2007;44:281-337 